\title{
Skin rejuvenation using cosmetic products containing growth factors, cytokines, and matrikines: a review of the literature
}

REVIEW

This article was published in the following Dove Press journal:

Clinical, Cosmetic and Investigational Dermatology

9 November 2016

Number of times this article has been viewed

\author{
Caroline Aldagl,* \\ Diana Nogueira Teixeira ${ }^{1, *}$ \\ Phillip S Leventhal ${ }^{2}$ \\ 'Merz Pharmaceuticals GmbH, \\ Frankfurt am Main, Germany; \\ ${ }^{2} 4$ Clinics, Paris, France \\ *These authors contributed equally to \\ this work
}

\begin{abstract}
Skin aging is primarily due to alterations in the dermal extracellular matrix, especially a decrease in collagen I content, fragmentation of collagen fibrils, and accumulation of amorphous elastin material, also known as elastosis. Growth factors and cytokines are included in several cosmetic products intended for skin rejuvenation because of their ability to promote collagen synthesis. Matrikines and matrikine-like peptides offer the advantage of growth factor-like activities but better skin penetration due to their much smaller molecular size. In this review, we summarize the commercially available products containing growth factors, cytokines, and matrikines for which there is evidence that they promote skin rejuvenation.
\end{abstract}

Keywords: cosmetics, skin, aging, growth factor, cytokine, matrikine

\section{Introduction}

Skin aging is a natural process caused by both intrinsic changes and extrinsic damage. ${ }^{1,2}$ Much of the change occurs in the dermis, which is mostly composed of a dense, collagen-rich extracellular matrix (ECM) that provides structure and support for the skin cells and confers tensile strength and firmness to the skin (Figure 1). ${ }^{1,2}$ Elastic fibers, which are made up of a cross-linked elastin core within fibrillin-based microfibrils, are key secondary components of the dermis that provide elasticity, resilience, and added tensile strength. ${ }^{3}$ Important changes also occur in the epidermis, most notably the accumulation of corneocytes, which causes the skin to take on a rough and dull appearance. ${ }^{1,4}$ In addition, reduced skin vasculature and structural changes to the subcutaneous tissue participate in skin aging.

Within the dermal ECM, aging is associated with a thickening of collagen fibrils and disorganization of total collagen content, mainly due to decreased collagen I synthesis and increased fibril fragmentation. ${ }^{1,2,5}$ In addition, skin aging is associated with increased levels of matrix metalloproteinases, which can break down collagen and elastin fibers, combined with impaired transforming growth factor (TGF)- $\beta$ signaling, which may reduce collagen deposition (Figure 2). 1,2,6

\section{Intrinsic versus extrinsic skin aging}

Although all aged skin shares common structural changes, intrinsically and extrinsically aged skin differs in several ways. Intrinsic aging occurs as a result of the natural chronological aging process, whereas extrinsic aging is brought about by environmental
Correspondence: Caroline Aldag

Merz Pharmaceuticals GmbH, Eckenheimer Landstraße 100, 60318

Frankfurt am Main, Germany

Tel +4969I503 4524

Fax $+4969 \quad 1503 \quad 1394$

Email caroline.aldag@merz.de. 


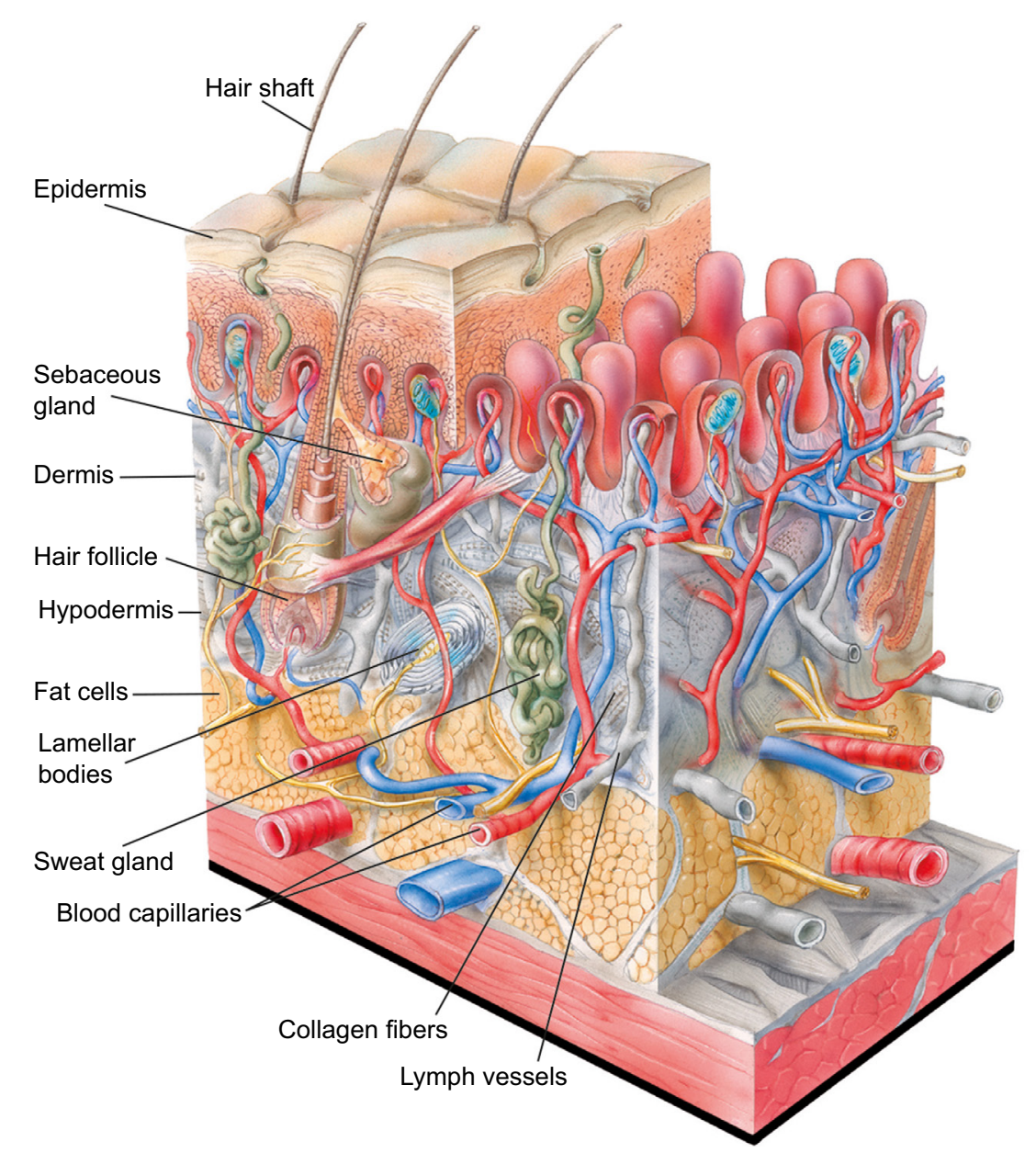

Figure I Structure of human skin.

Notes: The outer layer of the epidermis, the external layer of human skin, is made up primarily of corneocytes which provide a barrier function. Underlying the corneocytes are viable keratinocytes, which migrate outward and terminally differentiate to become corneocytes. The epidermis is organized into extensions called rete ridges that project between dermal papille (pink) into the underlying connective tissue. Underlying the epidermis is the dermis, which is primarily made up of collagen, elastin fibers, and other extracellular matrix components. Collagen and elastin fibers are synthesized by fibroblasts to provide tensile strength, firmness, and elasticity to the skin. The innermost layer of the skin, the hypodermis, is composed largely of fat cells, which helps provide structure to the skin. Blood capillaries, lymph vessels, sweat glands, sebaceous glands, hair follicles, and lamellar bodies lie within the dermis and hypodermis. Illustration: (c) www.julius-ecke.de; source: www.skin-care-forum.basf.com.

factors, especially sun exposure, also known as photoaging. ${ }^{1,4}$ Other common extrinsic factors are air pollution and cigarette smoke.

Intrinsic aging is associated with the natural deterioration of skin components, loss of cell turnover (senescence), and structural changes to subcutaneous tissue that occur over time. ${ }^{1}$ Intrinsically aged skin is characterized by laxity, fine wrinkling, and exaggerated expression lines, but it remains smooth and unblemished, with normal geometric patterns. ${ }^{1,2,7}$ In addition, intrinsically aged skin shows atrophy and flattened epidermal rete ridges, as well as a decreased ability to repair damage in conjunction with decreased numbers of fibroblasts and mast cells. In the dermal ECM, collagen fibrils and especially collagen I are lost.

Skin aged by extrinsic factors, in contrast to intrinsically aged skin, is characterized by dyschromia/mottled pigmentation (age spots), deep wrinkles, epidermal atrophy, and a leathery appearance. ${ }^{1,2,7}$ Elastosis, the accumulation of amorphous elastin material with reduced elasticity, is especially characteristic of extrinsically aged skin. ${ }^{1,2}$ In addition, collagen fibers in extrinsically aged skin become thickened, fragmented, and more soluble. In photoaged skin sections stained with hematoxylin and eosin, the accumulated abnormal collagen and elastin fibers appear as a blue mass over the Grenz zone, a narrow layer beneath the epidermis where new collagen is produced. ${ }^{8,9}$

Extrinsic factors are thought to age the skin by depleting antioxidants and increasing reactive oxygen species. ${ }^{10}$ Reactive oxygen species not only directly damage the skin structural proteins, but also induce overexpression of collagen-degrading matrix metalloproteinases and increase inflammation by the $\mathrm{NF}-\kappa \mathrm{B}$ pathway. ${ }^{10}$ In addition, 


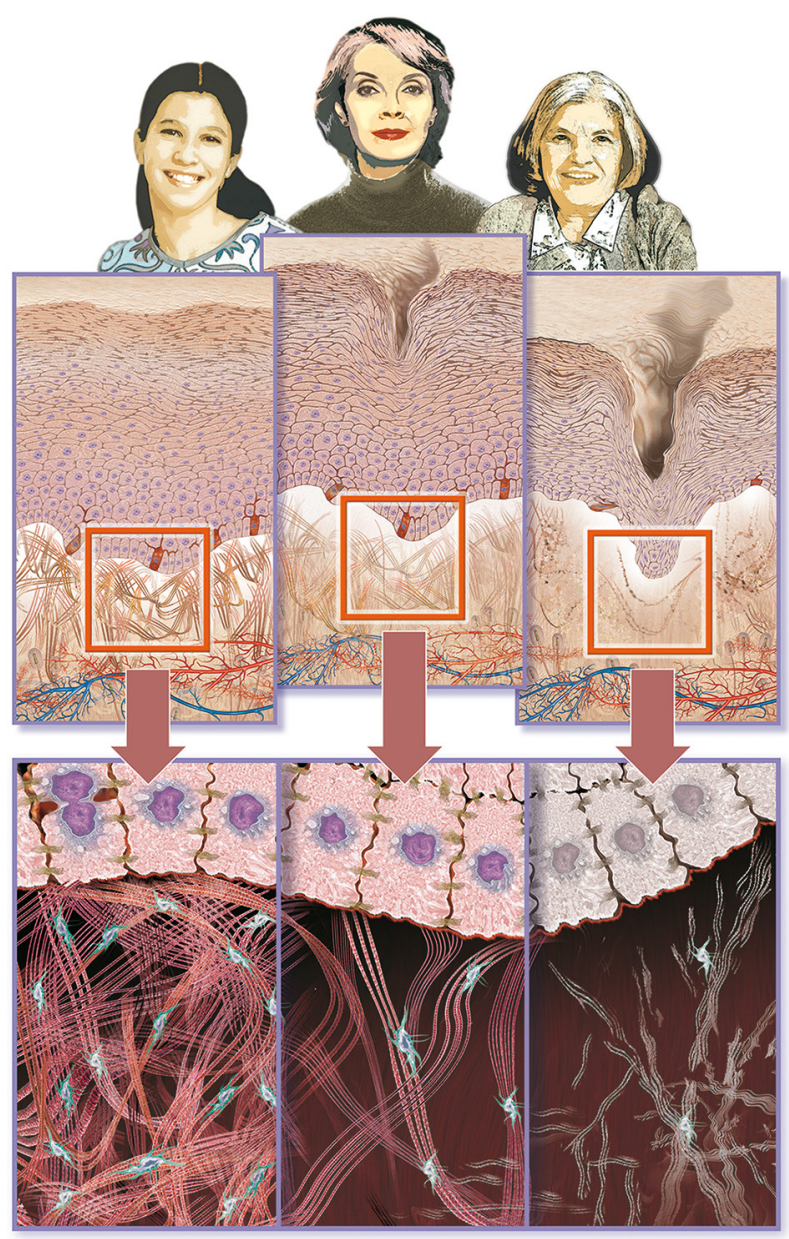

Figure 2 Changes in the skin with age.

Notes: As the skin ages, several changes occur in the epidermis and dermis. In the epidermis, corneocytes (terminally differentiated keratinocytes) accumulate, giving the skin a rough and dull appearance. In the dermis, the collagen content decreases and collagen and elastin fibers become disorganized and fragmented. This weakens the structure underlying the epidermis, leading to wrinkles. Illustration: (c) Kleinhans RED, source: www.skin-care-forum.basf.com.

ultraviolet light can directly damage the skin by causing nucleic acid strand breakage and by cross-linking and modifying the structural proteins and nucleic acids. ${ }^{4,11}$ Ultraviolet light also increases the accumulation of advanced glycation end-products, which can promote aging by several mechanisms. ${ }^{12}$

\section{Topical application of growth factors and cytokines for skin rejuvenation}

Growth factors and cytokines have been studied extensively in skin wound healing. ${ }^{13}$ Growth factors known to directly affect collagen biosynthesis include platelet-derived growth factor, vascular endothelial growth factor (VEGF), epidermal growth factor (EGF), granulocyte-colony stimulating factor, keratinocyte growth factor, and hepatocyte growth factor. ${ }^{13}$ Cytokines affecting collagen biosynthesis include TGF- $\beta$, interleukin (IL)-6, and IL-8. ${ }^{14-16}$
Although several studies indicate that topically applied growth factors and cytokines have been clinically shown to promote skin rejuvenation, their large molecular size (generally $>15,000 \mathrm{Da}$ ) limits their ability to penetrate the tightly packed stratum corneum. ${ }^{17}$ Molecules larger than $500 \mathrm{Da}$ generally cannot easily penetrate the stratum corneum to reach the viable keratinocytes in the stratum basale. One possible route of entry for larger molecules like growth factors and cytokines is through hair follicles, sweat glands, or compromised skin, for example, after microneedling or laser resurfacing. In addition, it may be possible to improve the penetration of growth factors and cytokines by chemical modification with lipophilic molecules. ${ }^{18}$

\section{Cosmetic products containing growth factors and cytokines for skin rejuvenation}

Several cosmetic products intended for skin rejuvenation contain growth factors and cytokines to promote collagen and elastin synthesis. ${ }^{13}$ We describe here products supported by clinical studies reported in peer-reviewed journal articles.

\section{Processed skin proteins (PSP $\left.{ }^{\circledR}\right)$ by Neocutis ${ }^{\circledR}$ (Merz North America, Inc., Raleigh, NC, USA)}

The PSP product line contains processed skin proteins, which are a mixture of growth factors and cytokines obtained as the lysate of cultured human fibroblasts. ${ }^{19}$ The studies performed so far suggest that the growth factors and cytokines in these PSP products reduce the appearance of skin aging, including fine lines and wrinkles.

In an initial case report, the effects of Bio-restorative Skin Cream, a PSP product, were described in two participants who had severe phototoxicity following 5-aminolevulinic acid (5-ALA) photodynamic therapy. ${ }^{20} 5$-ALA is a photosensitizer used to treat actinic keratosis, rosacea, inflammatory acne vulgaris, associated sebaceous disorders, and other skin conditions. ${ }^{21}$ Common adverse events of photodynamic skin therapy using 5-ALA include local and mostly minor erythema, edema, and crusting, which can last a week or more. Phototoxicity is rare, but can result in severe erythema lasting for weeks. The case report found that twice-daily application of Bio-restorative Skin Cream (for 3 or 7 days) helped return the skin to normal condition in the two participants.

This case report was followed up by a two-center, randomized, double-blind, split-face, placebo-controlled study in 20 women with facial wrinkling in the lateral canthal area. ${ }^{22} \mathrm{As}$ is common in placebo-controlled dermatologic studies, the 
vehicle (cream base) used to formulate the product was used as the control. Participants in the study were randomized to twice-daily application for 2 months of Bio-restorative Skin Cream or vehicle on the respective face halves. Threedimensional in vivo skin imaging was used to measure average roughness, mean roughness depth, maximum roughness depth, base roughness depth, maximum base roughness depth, and 10-point average roughness. In addition, investigators assessed wrinkles using the Rao-Goldman 5-point scale, which scores the wrinkles from 1 for no wrinkle to 5 for very deep wrinkles with redundant folds. ${ }^{23}$ Differences in roughness parameters and clinical assessments were compared by one-way analysis of variance (ANOVA) and were considered significant at $p \leq 0.05$. As measured by skin imaging, after 2 months, all roughness parameters significantly improved versus baseline with Bio-restorative Skin Cream, and three of six roughness parameters were significantly better with Bio-restorative Skin Cream than with vehicle. The investigators' clinical assessment of wrinkles, however, did not significantly differ between the Bio-restorative Skin Cream and the vehicle. In a separate publication based on the same study, according to investigators' visual assessments, periorbital and perioral wrinkles improved significantly at day 30 and day 60 versus baseline. ${ }^{24}$ At day 60 , periorbital wrinkle scores decreased by $17 \%$ and perioral wrinkle scores by $13 \%$. Also, chin texture improved significantly by $17 \%$ at day 60 . Finally, participants felt that Bio-restorative Skin Cream had improved their skin quality and appearance of wrinkles.

Further details of the effects of Bio-restorative Skin Cream were investigated in a single-center prospective study in 12 women with facial wrinkling in the lateral canthal area. ${ }^{25}$ Bio-restorative Skin Cream was applied twice daily for 6 months. Investigators assessed fine wrinkles in the periorbital and perioral areas on a scale of 0 for absent to 4 for very deep with redundant folds. They also took punch biopsies for microscopic analysis. According to the investigators, after 6 months, periorbital wrinkles decreased by $33 \%$ and perioral wrinkles by $25 \%$. Also, $55 \%$ of participants reported improved facial lines and wrinkles, 64\% reported improved facial skin texture, and $73 \%$ reported improved skin hydration. Light microscopy revealed a mild increase versus baseline in dermal fibroplasia and an associated reduction in elastosis, as well as increased numbers of fibrocytes and fibroblasts in $64 \%$ of participants and increased epidermal thickness in $55 \%$.

The effects of Lumiere ${ }^{\circledR}$ Bio-restorative Eye Cream, another PSP-containing product, were examined in a multicenter, open-label prospective study in 40 women with fine or deep wrinkles, visible dark areas, and slightly coarse or grainy lower eyelids. ${ }^{26}$ Investigators evaluated the skin color, texture, sagging, and wrinkles using 4-point scales. In addition, the participants answered a 10-item questionnaire about the quality of their periorbital skin, in which the answers were reported on 5-point scales. Differences in investigator and participant evaluations were assessed by repeated-measures ANOVA, with a $p$-value $\leq 0.05$ indicating a significant difference. Following twice-daily application of the product for 6 weeks, the investigators' clinical scores improved significantly versus baseline for skin texture (28\%), skin sagging (26\%), dark circles (15\%), and periorbital wrinkles (14\%). The participants' scores also improved significantly versus baseline for nine of ten parameters.

\section{TNS $^{\circledR}$ (SkinMedica, Carlsbad, CA, USA)}

The TNS product line includes TNS Recovery Complex ${ }^{\circledR}$ and TNS Essential Serum ${ }^{\circledR}$. TNS products contain conditioned medium obtained from neonatal foreskin fibroblast culture. ${ }^{27,28}$ The conditioned medium from these cells includes growth factors and cytokines that can promote angiogenesis (VEGF and hepatocyte growth factor), modulate inflammation (IL-6 and IL-8), and enhance ECM deposition (TGF- $\beta 1$ and platelet-derived growth factor-A)..$^{9,27,28}$ The currently available data indicate that the growth factors and cytokines in TNS may help improve the clinical appearance of aged skin.

A pilot study in 14 participants examined the effects of twice-daily application of TNS Recovery Complex for 60 days on photodamaged skin. ${ }^{9}$ Physicians measured photodamage on a 9-point scale. In addition, silicone impressions were made for optical profilometry, and punch biopsies were taken to assess Grenz zone and epidermal thickness. Differences were compared using two-tailed $t$-tests, with $p$-values $<0.05$ considered to indicate statistical significance. On the basis of investigators' evaluations, $11 / 14$ participants in the study showed improvement in at least one facial area. The periorbital region, in particular, showed a statistically significant improvement in clinically assessed photodamage $(p=0.0003)$. As shown by optical profilometry, the depth and number of textural irregularities or fine lines were found to have decreased significantly from baseline ( $p=0.0075)$, and ultrastructural evaluation indicated new collagen formation in the Grenz zone and thickening of the epidermis. In addition, eight of the participants felt that their wrinkles had improved, while 12 felt that their skin texture had improved.

TNS Recovery Complex was further studied in a single-center, randomized, double-blind, placebo-controlled study in 60 participants with mild-to-severe facial photodamage. ${ }^{28}$ In this study, the participants used a basic skin 
care regimen consisting of a cleanser and a moisturizer combined with twice-daily TNS Recovery Complex or a vehicle control for 6 months. Photodamage was assessed by optical profilometry using silicone impressions. Also, investigators assessed fine wrinkling, tactile roughness, telangiectasia, mottled pigmentation, and sallowness on a scale of 0 (absence of photodamage) to 4 (most severe photodamage). Differences were compared by paired $t$-test for profilometry and by two-sided Fisher's exact test for clinical assessments, with a $p$-value $\leq 0.05$ considered to indicate statistical significance. Optical profilometry revealed significantly improved facial lines and wrinkles at month 3 versus baseline in participants using TNS Recovery Complex. In addition, according to investigators' clinical assessments, the appearance of fine lines was significantly improved versus baseline for TNS Recovery Complex ( $p=0.012)$ but not for the vehicle control. However, measures of photodamage were not significantly different between TNS Recovery Complex and the vehicle control at month 6 , and only fine lines and texture shadows were significantly different at month 3 .

\section{ReGenica ${ }^{\circledR}$ (Histogen Aesthetics, San Diego, CA, USA)}

ReGenica is a third product line containing growth factors and cytokines from conditioned medium of fibroblasts $\left(\mathrm{MRCx}^{\mathrm{TM}}\right)$. The fibroblasts used to generate MRCx are grown on beads under hypoxic conditions to simulate the early embryonic environment prior to angiogenesis. ${ }^{29,30} \mathrm{MRCx}$ contains VEGF, keratinocyte growth factor, and IL-8, but not TGF- $\beta$.

In a proof-of-concept study including 49 participants who had been treated by laser resurfacing, a lotion containing MRCx produced a greater reduction in erythema and better re-epithelialization of the perioral and periocular areas than a placebo lotion. ${ }^{29}$ In a following split-face study in 42 participants, investigators assessed the effect of $\mathrm{MRCx}$, formulated as a gel, on transepidermal water loss, measured by Vapometer, and on erythema, edema, dryness, and peeling using a scale of 0 (none) to 4 (severe).$^{30}$ Erythema, edema, and crusting were also assessed by blinded observers using photographs and on the same 4-point scale. A $p$-value $\leq 0.05$ was considered to indicate statistical significance, although the statistical tests used were not described. The study found that MRCx, formulated as a gel, produced a dose-dependent improvement in crusting and significantly improved barrier function as measured by transepidermal water loss. ${ }^{30}$ In addition, biopsies, taken from three randomly selected subjects from each treatment group, showed that skin areas treated with the active gel had less leukocyte infiltration as well as more normal stratum germinativum and more normal organization of epidermal layers than the biopsies taken from skin areas treated with the vehicle control.

The effect of a 3-month application of ReGenica Replenishing Crème, applied every evening, combined with ReGenica Renew SPF 15, applied every morning, was examined in a study of 21 women with mild-to-moderate photodamage, fine lines, and wrinkles. ${ }^{31}$ ReGenica Replenishing Crème is a moisturizing cream containing $\mathrm{MRCx}$, and ReGenica Renew SPF 15 is a cream containing sunscreens and MRCX. Investigators assessed tactile roughness, visual texture, wrinkles, and blotchiness from 0 (none) to 4 (severe) and skin tone evenness, radiance, and translucency from 0 (worst possible results) to 4 (best possible results). Participants also completed questionnaires assessing overall satisfaction with the product and on a range of skin properties. Differences in investigator and participant assessments were compared by paired $t$-tests. Investigators' assessments of tactile roughness, visual texture, wrinkles, blotchiness, skin tone evenness, radiance, and translucency progressively and significantly improved at weeks 4,8 , and 12 versus baseline $(p<0.05)$. Participants also reported a similar statistically significant improvement in skin qualities at week 4 ( $p=0.034)$, week 8 ( $p=0.004)$, and week $12(p<0.001)$ versus the first post-baseline visit (at week 2). On the basis of these findings, the authors concluded that the topically applied growth factors and cytokines in MRCx promote recovery, improve skin barrier function, and may reduce inflammation in sun-damaged skin.

The effects of another MRCx product, ReGenica Revitalizing Eye Crème, were examined in a multicenter, open-label pilot study in 39 women of age $\geq 35$ years with wrinkles, uneven skin texture, puffiness, and lack of skin firmness in the lateral canthal and infraorbital areas. ${ }^{32}$ Using 10 -point scales, investigators assessed wrinkles, sagging, under-eye dark circles, sallowness, telangiectasia, and crepiness of the infraorbital area; wrinkles, sagging, dyschromia/mottled pigmentation, and crepiness of the lateral canthal area; and smooth texture, tightness, tone, brightness, and moistness of the infraorbital and lateral canthal areas. After applying the product twice daily for 60 days, participants reported improvements in infraorbital brightness, moistness, wrinkles, sallowness, crepiness, smooth texture, skin tightness, and skin tone. Investigators also found improvement in lateral canthal wrinkles, dyschromia/mottled pigmentation, skin tone, overall brightness, and moistness and that approximately 
two-thirds of participants were improved or much improved at days 14 and 60 compared to baseline.

\section{EGF serum (Bioeffect, Kopavogur, Iceland)}

EGF is a $6 \mathrm{kDa}$ polypeptide growth factor that has been of particular interest because of its ability to promote wound and ulcer healing when applied topically. ${ }^{33} \mathrm{~A}$ serum containing recombinant EGF produced in barley was examined in an open-label trial in 29 participants of age 30 years with photoaging. ${ }^{34}$ The participants completed a survey rating improvements in brown spots, red spots, age spots, and skin smoothness on a 5-point scale, and differences were compared by paired $t$-tests. The study found that twicedaily application of EGF serum for 3 months significantly improved brown spotting, skin texture, pore size, red spotting, and wrinkles versus baseline $(p<0.0002$ for all assessments).

\section{CRS with growth factor (Topix Pharmaceuticals, Amityville, NY, USA)}

Citrix ${ }^{\circledR}$ CRS Cell Rejuvenation System contains recombinant TGF- $\beta 1$, L-ascorbic acid, and extract from the plant Cimicifuga racemosa. This product was examined in a two-part, split-face, randomized study in women with facial wrinkling. ${ }^{35}$ In the first part of the study, facial halves of 11 women were randomized to twice-daily application of CRS or a similar cream lacking TGF- $\beta 1$ (VitC) for 3 months. In the second part, facial halves of 20 women were randomized to twice-daily application of TNS Recovery Complex or CRS for another 3 months. Blinded experts assessed photographs for wrinkles using a 5-point scale, and the participants completed questionnaires about changes in facial photoaging and satisfaction with the treatments. Differences were compared using two-tailed tests. The treatment was considered successful if the mean half-face wrinkle score showed a positive change or no worsening between baseline and the end of the study. According to investigator assessments, wrinkles showed "successful treatment" in 27 of 31 (87.1\%) participants who applied CRS, 14 of 19 (73.7\%) who applied TNS Recovery Complex, and seven of 12 (58.3\%) who applied VitC. In addition, 18 of 31 (58.1\%) participants who applied CRS, nine of 19 (47.4\%) who applied TNS Recovery Complex, and four of 12 (33.3\%) who applied VitC reported subjective improvement. The study also found significant improvements versus baseline in clinically assessed wrinkling ( $p=0.03$ ), but it did not report comparisons between regimens, so the effect of the cytokine TGF- $\beta 1$ was not clear.

\section{Cosmetic products containing matrikines and matrikine-like peptides for skin rejuvenation}

Matrikines are short peptides generated by proteolysis of ECM macromolecules that can modulate cell proliferation, migration, and apoptosis. ${ }^{36,37}$ Matrikines are widespread and, like growth factors, appear to have a variety of activities. For example, matrikines have been identified as markers of lung inflammation. ${ }^{38}$ In addition, like many growth factors, some matrikines can promote tumorigenesis, while others inhibit it. ${ }^{37,39-42}$ Matrikines have been of particular interest in skin rejuvenation because of their role in wound healing. ${ }^{37}$

Given their small molecular size, matrikines offer the possibility of better skin penetration than traditional growth factors and cytokines. Matrikine-like peptides are now included in several commercially available cosmetic formulations, although the effect of individual products has rarely been reported in the peer-reviewed literature. We, therefore, describe the existing clinical evidence for individual products and the in vitro and clinical evidence for single molecules included in currently marketed matrikinecontaining products.

\section{Glycine-histidine-lysine tripeptide (GHK)}

The tripeptide GHK is a matrikine that can complex with copper and, in skin, enhances ECM synthesis. ${ }^{36,43}$ GHK can penetrate the stratum corneum and accumulate to relevant concentrations in the skin. ${ }^{43}$ Application of a cream containing $\mathrm{Cu}-\mathrm{GHK}$, a complex of copper and $\mathrm{GHK}$, for 1 month was reported to increase collagen levels in the skin, as measured by immunohistochemistry. ${ }^{44}$

\section{Glycine-glutamate-lysine-glycine tetrapeptide (GEKG)}

GEKG, also known as tetrapeptide-21, is a matrikine whose activity was investigated in a series of in vitro studies and clinical assessments in participants with facial wrinkles. ${ }^{45}$ In the in vitro studies, the peptide increased procollagen secretion and collagen mRNA levels in human foreskin fibroblasts. In the first clinical assessment, which was a double-blind, randomized, placebo-controlled study in 10 healthy volunteers ( $>35$ years), daily application of a cream containing 50 ppm GEKG for 8 weeks significantly increased type I procollagen mRNA levels in the skin ( $p=0.02$ by Wilcoxon test). Further histochemical analysis revealed that GEKG, but not placebo, increased skin procollagen, hyaluronic acid, and fibronectin levels. GEKG improved point estimates of 
resilient distension and other elasticity assessments, although differences were not significant, possibly due to the small sample size. In a subsequent clinical assessment in the same report, 60 volunteers received twice-daily applications of 10 or $100 \mathrm{ppm}$ GEKG for 8 weeks. This portion of the study showed improved roughness parameters versus baseline at both 10 and 100 ppm GEKG. In a final clinical assessment in 30 volunteers, twice-daily application of 50 ppm GEKG for 4 or 8 weeks, but not a vehicle cream, improved periorbital wrinkling versus baseline. Collectively, the results indicated improvements in the skin quality, lines, and wrinkles, along with increased procollagen production.

\section{Lysine-threonine-threonine-lysine- serine pentapeptide (KTTKS)}

KTTKS is a matrikine derived from the proteolytic hydrolysis of collagen. ${ }^{46}$ This peptide promotes the production of ECM and the expression of fibronectin and collagen types I and III in vitro. ${ }^{46}$ It also stabilizes mRNAs that upregulate TGF- $\beta$, which may increase collagen production. ${ }^{47} \mathrm{~A}$ palmitoylated and more stable form of KTTKS (pal-KTTKS), also known as Matrixyl ${ }^{\circledR}$ or palmitoyl pentapeptide-3, has been reported to penetrate the stratum corneum ${ }^{48}$ and enhance collagen I secretion by fibroblasts in vitro. ${ }^{49}$ Pal-KTTKS has also been reported to significantly increase procollagen secretion and upregulate type I collagen and hyaluronic acid synthase-1 expression by human fibroblasts in vitro. ${ }^{45}$

The clinical outcome of an oil-in-water moisturizer containing pal-KTTKS was assessed in a double-blind, placebo-controlled, randomized, split-face study in 94 healthy Caucasian women with facial wrinkles. ${ }^{50}$ Total length of fine lines and wrinkles was assessed from digital photographs by computer algorithms. Also, blinded experts assessed improvements in skin texture, fine lines/wrinkles, age spots, dark circles under the eyes, and skin firmness using a scale of -4 (much worse) to +4 (much better). Differences in adjusted treatment means were compared by ANOVA using a mixed model, with a $p$-value $\leq 0.10$ indicating a significant difference. After twice-daily application for 8 and 12 weeks, fine line/wrinkle length was significantly lower with the palKTTKS cream than with a placebo cream, and clinically assessed fine lines and wrinkles were significantly better with the pal-KTTKS cream at weeks 8 and 12. In addition, skin texture at weeks 4 and 8 and age spots and dark circles at week 12 were significantly better with the pal-KTTKS cream. However, using a more standard definition of $p \leq 0.05$ to indicate statistical significance, only skin texture at 4 weeks and age spots at 12 weeks were significantly better.
No differences were found in skin texture or barrier function as measured by transepidermal water loss. Thus, although the in vitro studies suggest that KTTKS and pal-KTTKS increase collagen production, evidence of clinical relevance remains limited.

\section{Micro-protein complex (MPC ${ }^{\mathrm{TM}}$; Merz North America, Inc., Raleigh, NC, USA)}

MPC is a mixture of $N$-octanoyl-carnosine (an antioxidant) and the matrikines GEKG and palmitoyl-GHK. A recent report described a randomized, investigator-blinded study in which 133 healthy women were randomized 1:1:1 to MPC, PSP, or conditioned media growth factor. ${ }^{51}$ After a 2-week conditioning phase, during which the study participants washed their faces twice daily with a gentle cleanser and applied sun protection, the test products were applied for 6 months. An independent expert scored the severity of periorbital and perioral wrinkles using the Rao-Goldman 5-point visual scoring system and assessed the appearance of pores, tactile skin roughness, and additional indicators of skin aging using a 9-point visual analog scale. Skin elasticity and firmness were assessed by cutometry. In addition, facial wrinkle count, length, and area were analyzed in digital photos using a computer algorithm. Differences were analyzed by ANOVA, with $p$-values of $\leq 0.05$ considered to indicate statistical significance. According to the independent expert, periorbital wrinkles improved from baseline by an average of $23 \%$ at month $3(p<0.0001)$ and $33 \%$ at month $6(p<0.0001)$ in the MPC group. In addition, perioral wrinkles improved from baseline by an average $22 \%$ at month $3(p<0.001)$ and $31 \%$ at month $6(p<0.001)$. Similar improvements were observed in the PSP and conditioned media growth factor groups, and improvements were not statistically significantly different between the three groups. Mean periorbital wrinkle count significantly improved at each time point versus baseline for MPC and PSP $(p<0.05)$. The evaluator also judged that tactile skin roughness improved significantly from baseline by $51 \%$ at month 3 and $59 \%$ at month 6 ( $p<0.001$ for both) in the MPC group, and that pore appearance improved significantly from baseline at months 2,3 , and 6 ( $p \leq 0.001)$. For MPC users, quantitatively measured skin elasticity improved from baseline by an average of $20 \%$ at month $2(p<0.001)$, $13 \%$ at month $3(p=0.018)$, and $16 \%$ at month $6(p<0.001)$. In contrast, no significant improvements in skin elasticity were observed at any point in time with PSP or conditioned media growth factor. MPC also improved skin firmness by an average of $11 \%$ at month $3(p \leq 0.008)$ and $22 \%$ at month $6(p<0.001)$. 


\section{Conclusion}

The results of in vitro and clinical studies suggest that cosmetic products containing growth factors, cytokines, matrikines, or matrikine-like peptides can enhance the production of collagen and other ECM molecules and promote skin rejuvenation. Due to their small size, matrikines and matrikine-like peptides offer the promise of growth factorlike activities with improved skin penetration. However, data are limited. These products need to be further evaluated in well-designed, randomized trials to be able to draw firm conclusions about their clinical effects and mechanisms of action.

\section{Acknowledgments}

The authors would like to thank Frank Dreher and Uwe Aßmus for critically reading the manuscript. This work was funded by Merz Pharmaceuticals GmbH (Frankfurt am Main, Germany).

\section{Disclosure}

CA and DNT are employees of Merz Pharmaceuticals $\mathrm{GmbH}$, which sells products mentioned in this review. PSL is an employee of 4Clinics, which was paid for medical writing by Merz Pharmaceuticals GmbH. The authors report no other conflicts of interest in this work.

\section{References}

1. Baumann L. Skin ageing and its treatment.JPathol.2007;211(2):241-251.

2. Quan T, Fisher GJ. Role of age-associated alterations of the dermal extracellular matrix microenvironment in human skin aging: a minireview. Gerontology. 2015;61(5):427-434.

3. Sherratt MJ. Tissue elasticity and the ageing elastic fibre. Age (Dordr). 2009;31(4):305-325.

4. Rittie L, Fisher GJ. Natural and sun-induced aging of human skin. Cold Spring Harb Perspect Med. 2015;5(1):a015370.

5. Uitto J. The role of elastin and collagen in cutaneous aging: intrinsic aging versus photoexposure. J Drugs Dermatol. 2008;7(2 Suppl): S12-S16.

6. Sardy M. Role of matrix metalloproteinases in skin ageing. Connect Tissue Res. 2009;50(2):132-138.

7. Khavkin J, Ellis DA. Aging skin: histology, physiology, and pathology. Facial Plast Surg Clin North Am. 2011;19(2):229-234.

8. Montagna W, Kirchner S, Carlisle K. Histology of sun-damaged human skin. J Am Acad Dermatol. 1989;21(5 Pt 1):907-918.

9. Fitzpatrick RE, Rostan EF. Reversal of photodamage with topical growth factors: a pilot study. $J$ Cosmet Laser Ther. 2003;5(1):25-34.

10. Kammeyer A, Luiten RM. Oxidation events and skin aging. Ageing Res Rev. 2015;21:16-29.

11. Thurstan SA, Gibbs NK, Langton AK, Griffiths CE, Watson RE, Sherratt MJ. Chemical consequences of cutaneous photoageing. Chem Cent J. 2012;6(1):34.

12. Gkogkolou P, Bohm M. Advanced glycation end products: key players in skin aging? Dermatoendocrinol. 2012;4(3):259-270.

13. Fabi S, Sundaram H. The potential of topical and injectable growth factors and cytokines for skin rejuvenation. Facial Plast Surg. 2014;30(2):157-171.
14. Sproul EP, Argraves WS. A cytokine axis regulates elastin formation and degradation. Matrix Biol. 2013;32(2):86-94.

15. Uitto J, Kouba D. Cytokine modulation of extracellular matrix gene expression: relevance to fibrotic skin diseases. J Dermatol Sci. 2000;24(Suppl 1):S60-S69.

16. Verrecchia F, Mauviel A. Transforming growth factor-beta and fibrosis. World J Gastroenterol. 2007;13(22):3056-3062.

17. Mehta RC, Fitzpatrick RE. Endogenous growth factors as cosmeceuticals. Dermatol Ther. 2007;20(5):350-359.

18. Schaefer $\mathrm{H}$, Lademann J. The role of follicular penetration. A differential view. Skin Pharmacol Appl Skin Physiol. 2001;14(Suppl 1):23-27.

19. De Buys Roessingh AS, Hohlfeld J, Scaletta C, et al. Development, characterization, and use of a fetal skin cell bank for tissue engineering in wound healing. Cell Transplant. 2006;15(8-9):823-834.

20. Gold MH, Biron J. A novel skin cream containing a mixture of human growth factors and cytokines for the treatment of adverse events associated with photodynamic therapy. J Drugs Dermatol. 2006;5(8):796-798.

21. Nestor MS, Gold MH, Kauvar AN, et al. The use of photodynamic therapy in dermatology: results of a consensus conference. $J$ Drugs Dermatol. 2006;5(2):140-154.

22. Gold MH, Goldman MP, Biron J. Human growth factor and cytokine skin cream for facial skin rejuvenation as assessed by $3 \mathrm{D}$ in vivo optical skin imaging. J Drugs Dermatol. 2007;6(10):1018-1023.

23. Rao J, Ehrlich M, Goldman MP. Facial skin rejeuvenation with a novel topical compound containing transforming growth factor $\mathrm{b} 1$ and vitamin C. Cosmet Dermatol. 2004;17:705-713.

24. Gold MH, Goldman MP, Biron J. Efficacy of novel skin cream containing mixture of human growth factors and cytokines for skin rejuvenation. J Drugs Dermatol. 2007;6(2):197-201.

25. Hussain M, Phelps R, Goldberg DJ. Clinical, histologic, and ultrastructural changes after use of human growth factor and cytokine skin cream for the treatment of skin rejuvenation. $J$ Cosmet Laser Ther. 2008;10(2):104-109.

26. Lupo ML, Cohen JL, Rendon MI. Novel eye cream containing a mixture of human growth factors and cytokines for periorbital skin rejuvenation. J Drugs Dermatol. 2007;6(7):725-729.

27. Knighton DR, Ciresi K, Fiegel VD, Schumerth S, Butler E, Cerra F. Stimulation of repair in chronic, nonhealing, cutaneous ulcers using platelet-derived wound healing formula. Surg Gynecol Obstet. 1990;170(1):56-60.

28. Mehta RC, Smith SR, Grove GL, et al. Reduction in facial photodamage by a topical growth factor product. $J$ Drugs Dermatol. 2008;7(9):864-871.

29. Kellar RS, Hubka M, Rheins LA, Fisher G, Naughton GK. Hypoxic conditioned culture medium from fibroblasts grown under embryonic-like conditions supports healing following post-laser resurfacing. J Cosmet Dermatol. 2009;8(3):190-196.

30. Zimber MP, Mansbridge JN, Taylor M, et al. Human cell-conditioned media produced under embryonic-like conditions result in improved healing time after laser resurfacing. Aesthetic Plast Surg. 2012;36(2):431-437.

31. Bruce S, Karnik J, Dryer L, Burkholder D. Anti-aging proof of concept study: results and summary. J Drugs Dermatol. 2014;13(9):1074-1081.

32. Sundaram H, Gold M, Waldorf H, Lupo M, Nguyen VL, Karnik J. Pilot, multicenter, open-label evaluation of safety, tolerability and efficacy of a novel, topical multipotent growth factor formulation for the periorbital region. J Drugs Dermatol. 2015;14(12):1410-1417.

33. Hardwicke J, Schmaljohann D, Boyce D, Thomas D. Epidermal growth factor therapy and wound healing - past, present and future perspectives. Surgeon. 2008;6(3):172-177.

34. Schouest JM, Luu TK, Moy RL. Improved texture and appearance of human facial skin after daily topical application of barley produced, synthetic, human-like epidermal growth factor (EGF) serum. $J$ Drugs Dermatol. 2012;11(5):613-620.

35. Ehrlich M, Rao J, Pabby A, Goldman MP. Improvement in the appearance of wrinkles with topical transforming growth factor beta(1) and 1-ascorbic acid. Dermatol Surg. 2006;32(5):618-625. 
36. Maquart FX, Pasco S, Ramont L, Hornebeck W, Monboisse JC. An introduction to matrikines: extracellular matrix-derived peptides which regulate cell activity. Implication in tumor invasion. Crit Rev Oncol Hematol. 2004;49(3):199-202.

37. Tran KT, Lamb P, Deng JS. Matrikines and matricryptins: implications for cutaneous cancers and skin repair. J Dermatol Sci. 2005;40(1):11-20.

38. Abdul Roda M, Fernstrand AM, Redegeld FA, Blalock JE, Gaggar A, Folkerts G. The matrikine PGP as a potential biomarker in COPD. Am J Physiol Lung Cell Mol Physiol. 2015;308(11):L1095-L1101.

39. Monboisse JC, Oudart JB, Ramont L, Brassart-Pasco S, Maquart FX. Matrikines from basement membrane collagens: a new anti-cancer strategy. Biochim Biophys Acta. 2014;1840(8):2589-2598.

40. Brassart-Pasco S, Senechal K, Thevenard J, et al. Tetrastatin, the NC1 domain of the alpha4(IV) collagen chain: a novel potent anti-tumor matrikine. PLoS One. 2012;7(4):e29587.

41. Williams KE, Fulford LA, Albig AR. Lumican reduces tumor growth via induction of fas-mediated endothelial cell apoptosis. Cancer Microenviron. 2010;4(1):115-126.

42. Ramont L, Brassart-Pasco S, Thevenard J, et al. The NC1 domain of type XIX collagen inhibits in vivo melanoma growth. Mol Cancer Ther. 2007;6(2):506-514.

43. Pickart L, Vasquez-Soltero JM, Margolina A. The human tripeptide $\mathrm{GHK}-\mathrm{Cu}$ in prevention of oxidative stress and degenerative conditions of aging: implications for cognitive health. Oxid Med Cell Longev. 2012;2012:324832.
44. Abdulghani AA, Sherr A, Shirin S, et al. Effects of topical creams containing vitamin $\mathrm{C}$, a copper-binding peptide cream and melatonin compared with tretinoin on the ultrastructure of normal skin - a pilot clinical, histologic, and ultrastructural study. Dis Manag Clin Outcome. 1998;1(4):136-141.

45. Farwick M, Grether-Beck S, Marini A, et al. Bioactive tetrapeptide GEKG boosts extracellular matrix formation: in vitro and in vivo molecular and clinical proof. Exp Dermatol. 2011;20(7):602-604.

46. Abu Samah NH, Heard CM. Topically applied KTTKS: a review. Int J Cosmet Sci. 2011;33(6):483-490.

47. Tsai WC, Hsu CC, Chung CY, Lin MS, Li SL, Pang JH. The pentapeptide KTTKS promoting the expressions of type I collagen and transforming growth factor-beta of tendon cells. J Orthop Res. 2007;25(12):1629-1634.

48. Choi YL, Park EJ, Kim E, Na DH, Shin YH. Dermal stability and in vitro skin permeation of collagen pentapeptides (KTTKS and palmitoylKTTKS). Biomol Ther (Seoul). 2014;22(4):321-327.

49. Jones RR, Castelletto V, Connon CJ, Hamley IW. Collagen stimulating effect of peptide amphiphile C16-KTTKS on human fibroblasts. Mol Pharm. 2013;10(3):1063-1069.

50. Robinson LR, Fitzgerald NC, Doughty DG, Dawes NC, Berge CA, Bissett DL. Topical palmitoyl pentapeptide provides improvement in photoaged human facial skin. Int J Cosmet Sci. 2005;27(3):155-160.

51. Dreher F. A novel matrikine-like micro-protein complex (MPC) technology for topical skin rejuvenation. J Drugs Dermatol. 2016;15(4):611-618.
Clinical, Cosmetic and Investigational Dermatology

\section{Publish your work in this journal}

Clinical, Cosmetic and Investigational Dermatology is an international, peer-reviewed, open access, online journal that focuses on the latest clinical and experimental research in all aspects of skin disease and cosmetic interventions. This journal is included on PubMed. The manuscript management system is completely online

\section{Dovepress}

and includes a very quick and fair peer-review system, which is all easy to use. Visit http://www.dovepress.com/testimonials.php to read real quotes from published authors 